\title{
Prognostic significance of FOXM1 expression and antitumor effect of FOXM1 inhibition in synovial sarcomas
}

Akira Maekawa ${ }^{1}$, Kenichi Kohashi ${ }^{1}$, Masaaki Kuda ${ }^{1}$, Kunio lura' ${ }^{1}$, Takeaki Ishii ${ }^{1}$, Makoto Endo ${ }^{2}$, Tetsuya Nakatsura ${ }^{3}$, Yukihide Iwamoto ${ }^{2}$ and Yoshinao Oda ${ }^{1 *}$

\begin{abstract}
Background: Synovial sarcoma (SS) is a soft tissue sarcoma of unknown histogenesis. Most metastatic or unresectable cases are incurable. Novel antitumor agents and precise prognostication are needed for SS patients. The protein forkhead box M1 (FOXM1), which belongs to the FOX family of transcription factors, is considered to be an independent predictor of poor survival in many cancers and sarcomas, but the prognostic implications and oncogenic roles of FOXM1 in SS are poorly understood. Here we examined the correlation between FOXM1 expression and clinicopathologic and prognostic factors, and we investigated the efficacy of FOXM1 target therapy in SS cases.
\end{abstract}

Methods: Immunohistochemical study of 106 tumor specimens was conducted to evaluate their immunohistochemical expression of FOXM1. An in vitro study examined the antitumor effect of the FOXM1 inhibitor thiostrepton and small interference RNA (siRNA) on two SS cell lines. We also assessed the efficacy of the combined use of doxorubicin (DOX) and thiostrepton.

Results: Univariate and multivariate analyses revealed that FOXM1 expression was associated with poor prognosis in SS. The cDNA microarray analysis using clinical samples revealed that the expression of cell cycle-associated genes was correlated with FOXM1 expression. FOXM1 inhibition by thiostrepton showed significant antitumor activity on the SS cell lines in vitro. FOXM1 interruption by siRNA increased the chemosensitivity for DOX in both SS cell lines.

Conclusion: FOXM1 expression is a novel biomarker, and its inhibition is a potential treatment option for SS.

Keywords: Forkhead box M1 (FOXM1), Synovial sarcoma, Thiostrepton

\section{Background}

Synovial sarcoma (SS) is a soft tissue sarcoma of unknown histogenesis, occurring most frequently in adolescents and young adults. It is mainly classified into three histological subtypes: the biphasic type composed of both epithelial and spindle-cell components, the monophasic fibrous type composed of either an epithelial or spindle-cell component, and the poorly differentiated type [1]. SS has a genetic event, the $\mathrm{t}(\mathrm{X}: 18)$ translocation-mediated fusion of the SS18 gene on chromosome18q 11 to either SSX1,

\footnotetext{
* Correspondence: oda@surgpath.med.kyushu-u.ac.jp

${ }^{1}$ Department of Anatomic Pathology, Graduate School of Medical Sciences, Kyushu University, 3-1-1 MaidashiHigashi-ku, Fukuoka 812-8582, Japan Full list of author information is available at the end of the article
}

SSX2, or rarely SSX4 gene located on chromosome (p11.2;q11.2) [2]. The reported 5-year survival rates of patients with SS range from 64 to $77 \%$ [3-6]. Most metastatic or relapsed diseases remain incurable, Efficacy of adjuvant chemotherapy in resected primary SS cases is still unclear [6]. Novel antitumor agents and precise prognostication are essential to improve the survival of SS patients.

The protein forkhead box M1 (FOXM1), a member of the FOX family of transcription factors, is widely expressed in embryonic tissues $[7,8]$. Terminally differentiated nonproliferating tissues display relatively low levels of FOXM1 expression [9]. FOXM1 regulates a wide spectrum of tumor progression processes [10]. 
Increased levels of FOXM1 expression have been detected in many different types of human cancer [11-21] and sarcoma such as rhabdomyosarcoma [22], Ewing sarcoma [23], malignant peripheral nerve sheath tumor [24], and osteosarcoma [25, 26]. Silencing FOXM1 expression suppressed the proliferation of both cancer [16, 18, 22] and sarcoma cell lines $[22,26]$. In various carcinoma cell lines, FOXM1 was also involved in resistance to chemotherapy drugs such as doxorubicin (DOX) [27], which is a frequently used antitumor agent against soft tissue sarcoma. The inhibition of FOXM1 may thus have the potential to be a therapeutic target for many malignancies. Both the prognostic impact of FOXM1 expression and the effectiveness of FOXM1 inhibition in SS remain to be clarified.

Here, we conducted a clinicopathologic and prognostic analysis of the FOXM1 expression in a series of 106 clinical specimens of SS, and a cDNA microarray analysis in 11 frozen samples. Using small interference RNA (siRNA), we then tested the involvement of FOXM1 in tumor progression and the acquisition of drug resistance. We also tested the efficacy of the combined use of DOX and FOXM1 inhibition (by thiostrepton and siRNA) in SS cell lines in vitro.

\section{Methods}

\section{Patients and clinical information}

We examined 106 SS patients registered in the Department of Anatomic Pathology, Graduate School of Medical Sciences, Kyushu University, Japan, between 1990 and 2014. Each tumor had been classified histologically into the monophasic fibrous, biphasic, or poorly differentiated type according to the most recent World Health Organization classification [28] including the examination of SS18-SSX1 and SS18-SSX2 fusion transcripts. The extents of necrosis and mitosis were evaluated according to the French Federation of Cancer Centers (FNCLCC) grading system [28]. For the staging of the primary tumors, the latest American Joint Committee on Cancer (AJCC) staging system was used [29]. Surgical margins were available in 49 patients (39 cases, wide marginal resection; 9 cases, marginal resection; 2 cases, intralegional resection).

We also analyzed the FOXM1 expression and EFS rate in 19 patients who had undergone pre- or/and postoperative chemotherapy. Eighteen of these patients had a wide margin; one patient underwent surgical resection, and one patient was treated with heavy ion irradiation. Most of the chemotherapy regimens were a single use of DOX or a combination of DOX and ifosfamide. This study was conducted in accordance with the principles embodied in the Declaration of Helsinki, and was approved by the Ethics Committee of Kyushu University (No. 26-49).

\section{Cell lines}

We analyzed SYO-1 [30] was established by Dr. Kawai and HS-SY-II [31] was established by Dr. Sonobe as synovial sarcoma cell lines. These cell lines were authenticated by confirming the expression of pathognomonic SS18-SSX fusion genes by reverse transcriptase polymerase chain reaction (RT-PCR) in October 2012. All cell lines were cultured in Dulbecco's modified Eagle's medium (DMEM) supplemented with $10 \%$ fetal bovine serum (FBS) plus penicillin.

\section{Drugs}

Doxorubicin (DOX) was obtained from Cell Signaling Technology (Tokyo), and Thiostrepton was obtained from Millipore/EMD (Billerica, MA, USA). Both drugs were dissolved in DMSO (Sigma-Aldrich, St. Louis, MO) and were used at the indicated concentrations.

\section{Detection of fusion gene transcripts}

We performed an SS18-SSX fusion assay based on the reported primers [32] that specifically amplify the fusion gene transcripts of SS18-SSX1 and SS18-SSX2. Each PCR product $(5 \mu \mathrm{L})$ was loaded onto a $2 \%$ agarose gel with ethidium bromide and visualized under UV illumination. The PCR products were also evaluated by direct sequence analysis using the Big-Dye terminator method (version 1.1; Applied Biosystems, Foster City, CA) to confirm the breakpoints of fusion transcripts.

\section{Immunohistochemical study}

All 106 formalin-fixed, paraffin-embedded specimens were cut at $3 \mu \mathrm{m}$. Antigen retrieval was carried out by boiling the slides with Target retrieval solution (TRS; Dako, Carpinteria, CA). The primary antibody was monoclonal anti-human FOXM1 antibody (R\&D Systems, Minneapolis, MN) diluted at 1:300. All immune complexes were visualized by the EnVision ${ }^{\mathrm{Tm}}$ System Detection system (Dako).

We used biopsy specimens for the evaluation of FOXM1 expression if the patients received pre-operative chemotherapy. For FOXM1, immunoreactivity was defined as cells showing nuclear staining with/without cytoplasmic staining patterns in the tumor tissue with minimal background staining. Tumors with a strong staining intensity in $>10 \%$ of the tumor cells were recorded as having positive immunoreactivity for FOXM1 based on a reported method $[11,12]$. The serial sections were also immunostained with anti-Ki-67 antibody (M 7240, 1:100; Dako Glostrup, Denmark) using the standard procedure. The Ki-67-labeling index was calculated as described [33].

\section{Gene expression profiling of cDNA micro array}

We conducted cDNA micro array analysis in 11 frozen samples obtained from primary SS cases. For the Oligo 
DNA microarray analysis, 3D-Gene Human Oligo chip $25 \mathrm{k}$ (Toray Industries, Tokyo) was used (25,370 distinct genes). For efficient hybridization, this microarray has three dimensions and is constructed with a well as the space between the probes and cylinder-stems with 70-mer oligonucleotide probes on the top. Total RNA was labeled with Cy5 using the Amino Allyl MessageAMP ${ }^{\text {tw }}$ II aRNA Amplification Kit (Applied Biosystems). The Cy5-labeled aRNA pools and hybridization buffer, and hybridized for $16 \mathrm{~h}$.
The hybridization was performed using the supplier's protocols (www.3d-gene.com). Hybridization signals were scanned using a ScanArray Express Scanner (PerkinElmer, San Jose, CA), and processed by GenePixPro software, ver. 5.0 (Molecular Devices, Sunnyvale, CA). The raw data of each spot was normalized by subtraction with a mean intensity of the background signal determined by all blank spots' signal intensities of $95 \%$ confidence intervals (CI). The signals detected for each gene were normalized by

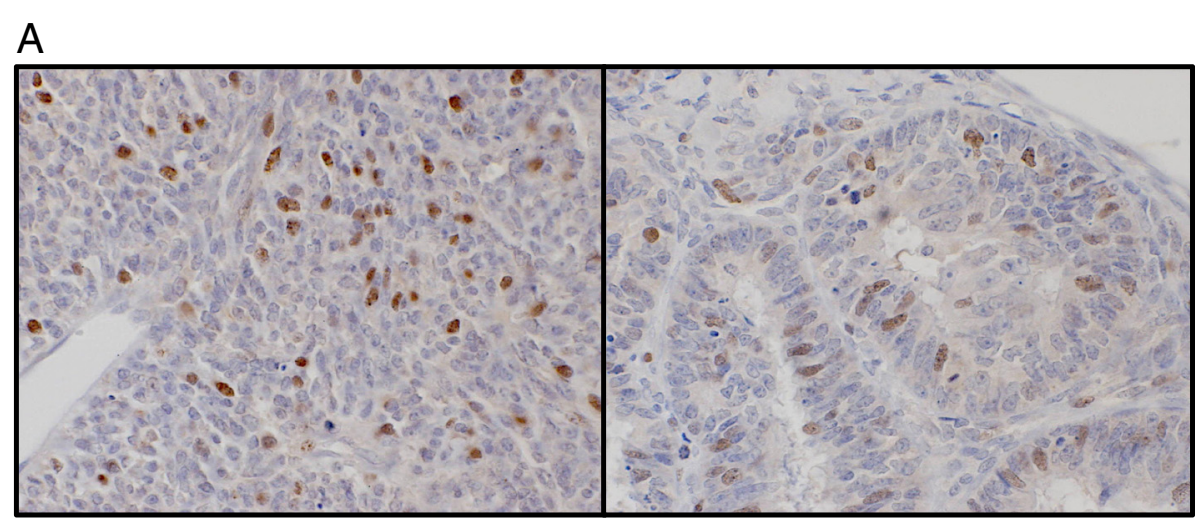

B
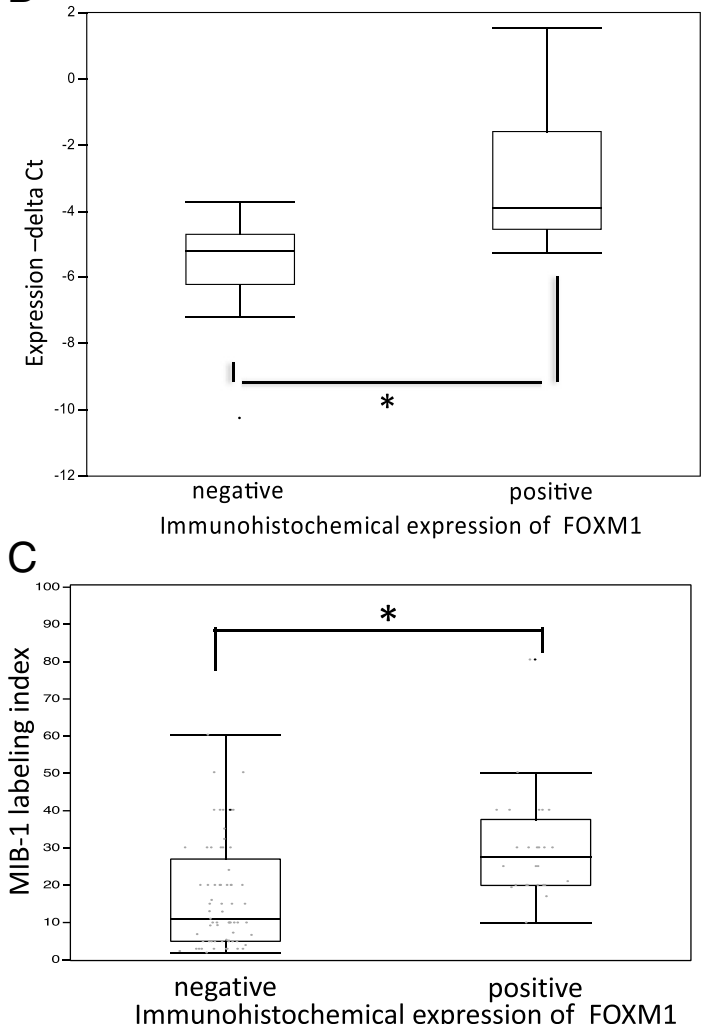

Fig. 1 a Immunohistochemical results for FOXM1: Monophasic fibrous type (left) and biphasic type (right). Immunostaining for antibody was recognized in the nuclei. b Quantitative RT-PCR and immunohistochemical stain for FOXM1 in clinical samples. The RT-PCR values are plotted as: $1 \times$ (cross threshold [Ct] FOXM1 - Ct GAPDH). High Ct values indicate high gene expression, and vice versa. The results are the means \pm SD. ${ }^{*} P<0.05$ by $t$-test. $\mathbf{c}$ Correlation of FOXM1 expression and MIB-1 labeling index in clinical specimens. The MIB-1 labeling index was significantly high in the FOXM1 expression cases. The results are means \pm SD. ${ }^{*} P<0.05$ by $t$-test 
Table 1 Clinicopathologic parameters, FOXM1 expression and survival analysis

\begin{tabular}{|c|c|c|c|c|c|c|c|}
\hline \multirow[t]{2}{*}{ Variable } & \multirow{2}{*}{$\begin{array}{l}\text { No. of } \\
\text { patients }\end{array}$} & \multirow[t]{2}{*}{ Analyzed groups } & \multicolumn{2}{|l|}{$P$-value } & \multicolumn{3}{|l|}{ FOXM1 } \\
\hline & & & OS & EFS & Positive & Negative & $P$-value \\
\hline \multicolumn{8}{|l|}{ Sex } \\
\hline Male & 44 & & & & 13 & 31 & \\
\hline Female & 62 & Male vs. female & $0.0028^{*}$ & 0.3047 & 17 & 45 & 0.811 \\
\hline \multicolumn{8}{|l|}{ Age } \\
\hline$<20$ & 19 & & & & 4 & 15 & \\
\hline$\geq 20$ & 87 & $20<$ vs. $\geq 20$ & $0.0189^{*}$ & 0.2499 & 26 & 61 & 0.4279 \\
\hline \multicolumn{8}{|l|}{ Chemotherapy } \\
\hline Yes & 25 & & & & 6 & 19 & \\
\hline No & 25 & Yes vs. No & 0.3431 & 0.8037 & 4 & 21 & 0.4783 \\
\hline N.A. & 56 & & & & & & \\
\hline \multicolumn{8}{|l|}{ Fusion gene type } \\
\hline SS18-SSX1 & 30 & & & & 11 & 19 & \\
\hline SS18-SSX2 & 14 & SSX1 vs. SSX2 & 0.6271 & 0.8581 & 3 & 11 & 0.3736 \\
\hline N.A. & 62 & & & & & & \\
\hline \multicolumn{8}{|l|}{ Depth } \\
\hline Superficial & 13 & & & & 5 & 8 & \\
\hline Deep & 91 & Deep vs. Superficial & 0.4441 & 0.9057 & 25 & 66 & 0.4243 \\
\hline N.A. & 2 & & & & & & \\
\hline \multicolumn{8}{|l|}{ Size,cm } \\
\hline$<5$ & 40 & & & & 8 & 32 & \\
\hline $5 \geq$ & 62 & $<5$ vs. $5 \leq$ & $0.0012^{*}$ & $0.0335^{*}$ & 22 & 40 & 0.0885 \\
\hline N.A. & 4 & & & & & & \\
\hline \multicolumn{8}{|c|}{ Histological subtype } \\
\hline Mono & 69 & & & & 22 & 47 & \\
\hline $\mathrm{Bi}$ & 26 & Mono vs. bi & 0.4225 & 0.9701 & 3 & 23 & $0.0335^{*}$ \\
\hline Poor & 3 & & & & 1 & 2 & \\
\hline Undetermined & 8 & & & & & & \\
\hline \multicolumn{8}{|l|}{ Necrosis } \\
\hline None & 56 & & & & 13 & 43 & \\
\hline$\leq 50 \%$ & 26 & Necrosis (+) vs. $(-)$ & $<0.001^{*}$ & $0.0012^{*}$ & 9 & 17 & 0.1526 \\
\hline$>50 \%$ & 15 & & & & 6 & 9 & \\
\hline N.A. & 9 & & & & & & \\
\hline \multicolumn{8}{|l|}{ Mitotic count } \\
\hline$\geq 10 / 10 \mathrm{HPF}$ & 70 & & & & 12 & 58 & \\
\hline
\end{tabular}

N.A.

9

Mitotic count

$\begin{array}{llllll} & & & 12 & 58 & \\ & & & 12 & & \\ & 0.0344^{*} & 0.0056^{*} & 17 & 14 & 0.0002^{*}\end{array}$

N.A.

AJCC stage

II

N.A.

10

FNCLCC

|| vs. III

III vs. IV

$0.0304^{*}$

0.3734

$<0.001^{*}$

3

0

0.72
0.6579 
Table 1 Clinicopathologic parameters, FOXM1 expression and survival analysis (Continued)

\begin{tabular}{lcccc}
\hline 3 & 23 & 2 vs. 3 & $<0.001^{*}$ & $<0.001^{*}$ \\
N.A. & 14 & & 10.123 \\
FOXM1 & 30 & & \\
Positive & 76 & $0.0128^{*}$ & $0.0043^{*}$ \\
Negative & Positive vs. negative & - \\
$\begin{array}{l}\text { AJCC American Joint Committee on Cancer, Bi biphasic synovial sarcoma, EFS event-free survival, FNCLCC French Federation of Cancer Centers, HPF high-power } \\
\text { fields, Mono monophasic synovial sarcoma, Poor poorly differentiated synovial sarcoma, NA not available, OS overall survival } \\
{ }^{*} P<0.05 \text { by log-rank test or chi-square test }\end{array}$
\end{tabular}

the global normalization method (the median of the detected signal intensity was adjusted to 25). Genes correlated with FOXM1 were extracted by the hierarchal clustering method. We defined "correlate" as a correlation coefficient $(\mathrm{CC})>0.828$. We also conducted a gene ontology (GO) analysis using the Gene Ontology Consortium (http://geneontology.org/).

\section{SiRNA}

Both SYO-1 and HS-SY-II cells were transfected with OnTarget plus Smart Pool siRNAs FOXM1 (Dhamacon, CO, USA) and On-Target plus Non-targeting Pool (Dhamacon, CO, USA) as a control, using Lipofectamine RNA imax (Invitrogen, MA, USA) according to the manufacturer's protocols. The introduction of the siRNA for FOXM1 was confirmed by qRT-PCR and immunoblotting.

\section{TaqMan PCR to detect mRNA quantity of FOXM1}

Total RNA was extracted using miRNeasy Mini kit (Qiagen). Five micrograms of RNA from each sample were reverse-transcribed using Quantitect Reverse Transcription Kit (Qiagen) in order to prepare first-strand cDNA. We performed a quantitative RT-PCR for FOXM1 and analyzed using TaqMan assay reagents (FOXM1 Hs00170471_m1.; GAPDH Hs99999905_m1.; Applied Biosystems) and an ABI Prism 7700 Sequence Detection system (Applied Biosystems). RNA was obtained from 23 frozen samples and cell lines, using Qiagen mi RNA extraction kit (Qiagen, Venlo, Netherlands). The RNA extraction and PCR reaction were carried out according to the manufacturer's protocol. The obtained data were standardized using the data of the housekeeping gene GAPDH. All of the reactions for each sample were performed in at least triplicate. The data were averaged from the values obtained in each reaction.

\section{Western blot}

The cells were washed twice with ice-cold phosphatebuffered saline (PBS), scraped, and collected in a microcentrifuge tube. Whole cell lysates were prepared from the cell lines. Anti-FOXM1 (1:200 dilution) antibody (R\&D Systems). Anti-human actin mouse monoclonal antibody (1:5000; Millipore) was used as a loading control. The subsequent Western blot procedure was performed as described [33].

\section{Cell viability}

Cell viability was assessed by an MTT assay using the Cell Counting Kit 8 (CCK-8, Dojindo Molecular Technologies, Rockville, MD) according to the manufacturer's instructions and as described [33]. The absorbance at $450 \mathrm{~nm}$ was measured by a microplate reader (Model 680, BioRad Laboratories) by spectrophotometry at $450 \mathrm{~nm}$.

\section{Drug treatment and cell proliferation assay of the transfected cell lines}

After 24-h siRNA transfection, the transfected cells were seeded at 5000 cells per well in 96-well plates. For the chemosensitivity assay, various concentrations of DOX were added to the medium after 12-h incubation. After another incubation for $72 \mathrm{~h}$, the number of viable cells in each well was measured.

For the proliferation assay, the number of viable cells in each well was measured at $36,48,72$, and $96 \mathrm{~h}$ after transfection. Assays were conducted in triplicate and were repeated at least three times in separate experiments.

\section{Drug treatment and cell proliferation assay}

SYO-1 and HS-SY-II cells were plated on 96-well plates at a concentration of 5000 cells per well in serumcontaining growth medium. After a 12-h incubation, cells were treated with carrier alone $(0.01 \%$ DMSO) as non-treated control or with various concentrations of DOX, thiostrepton, or thiostrepton + DOX for another $72 \mathrm{~h}$. The resulting data are reported as the percentage of cell viability in comparison to that of the respective non-treated control group (100\%). Assays were conducted in triplicate and were repeated at least three times in separate experiments.

\section{Statistical analysis}

We used the chi-square test and the $t$-test as appropriate to evaluate associations between two variables. The Steel-Dwass multiple comparison test was applied to compare the data of more than two groups. The survival correlations are illustrated with Kaplan-Meier curves, 
and survival analyses were performed using the log-rank test. In the multivariate analysis, a Cox proportional hazards model was used to independent examine factors. Two-sided $P$-values $<0.05$ were considered significant.

\section{Results}

\section{Prognostic significance of FOXM1 expression in synovial} sarcoma patients

Survival data were available for overall survival (OS) in 103 patients, who were followed-up from 1 to 278 months (median, 85 months). The 5-year OS rate was $62 \%$. Data were available for event-free survival (EFS) in 70 patients, who had a follow-up ranging from 4 to 278 months (median, 81 months) and whose 5-year EFS rate was $56 \%$.

Immunohistochemically, the positive expression of FOXM1 was recognized in 28 of the 106 SS cases. SS cells showed nuclear staining for FOXM1 antibodies (Fig. 1a). The mRNA expressions of the samples that were immunohistochemically positive for FOXM1 showed a significantly higher mean cross threshold (mean, $-2.95 \pm 2.20$ ) compared to the immunohistochemically negative expression samples (mean, $-5.64 \pm 1.63 ; P=0.002$ ) (Fig. 1b). In addition, the MIB-1 labeling index was significantly higher in the FOXM1 expression cases (positive $29.3 \pm 13.5$ vs. negative $16.9 \pm 14.0, P=0.0002$ ) (Fig. 1c).

The clinicopathologic data and the results of the survival analysis of all 106 patients are summarized in Table 1. We evaluated the correlations between the immunohistochemical results and over-all (OS) or eventfree (EFS) survival. Immunopositivity for FOXM1 was found to be a significant risk factor for adverse prognosis (OS and EFS). The Kaplan-Meier survival curves for OS and EFS are shown in Fig. 2a,b. Among the 19 patients treated with chemotherapy, the 3 FOXM1 expression cases had poor prognoses in EFS (Fig. 2c).

The following clinicopathologic variables were also revealed to be significantly associated with poor prognosis: large tumor size $(>5 \mathrm{~cm})$, the presence of tumor necrosis, high mitotic activity (>10/10 HPF), advanced AJCC stage (II vs. III and III vs. VI), sex (female) and age ( $>20$ year). The associations of the clinicopathological parameters with FOXM1 are shown in Table 1. FOXM1 was significantly associated with the histological subtype (monophasic fibrous variant) and high mitotic activity (>10/10 HPF).

We also conducted a multivariate analysis for FOXM1 with clinicopathologic variables adjusted by sex, age and AJCC surgical stage (II, III and IV) that were related to poor prognosis in the univariate analysis. We excluded tumor depth, tumor size, mitotic count, necrosis and FNCLCC histological grade, because the AJCC surgical stage is derived from these. The multivariate analysis revealed that FOXM1 expression and AJCC staging are significantly correlated with overall survival (Table 2).

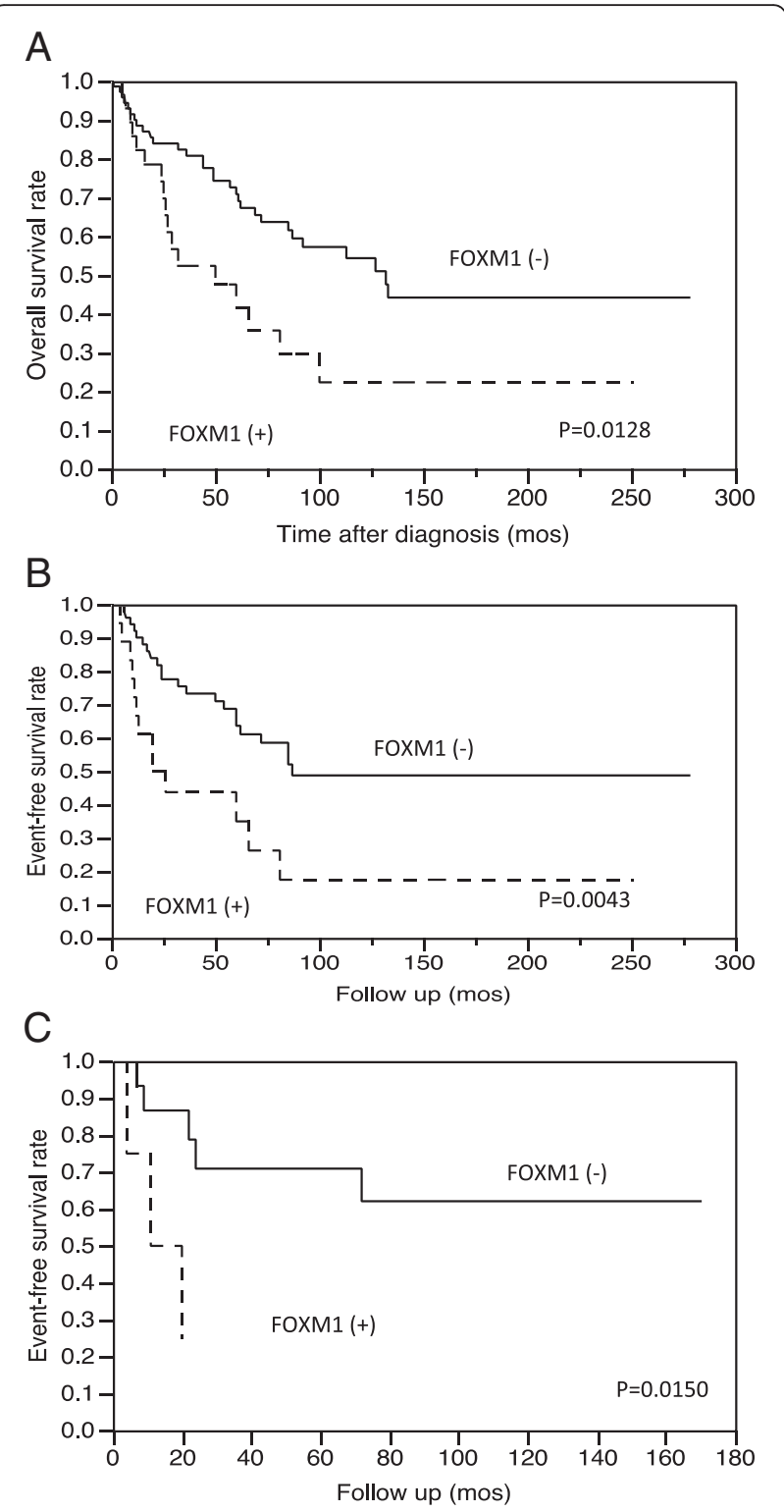

Fig. 2 Kaplan-Meier survival curves for the patients' overall survival (OS) and event-free survival (EFS) according to the results of the immunohistochemical study for FOXM1: (a) OS, (b) EFS, and (c) EFS for the 19 patients treated with chemotherapy

Table 2 Multivariate analysis for immunohistochemical and clinicopathological parameters

\begin{tabular}{ll}
\hline Multivariate analysis & \\
\hline Variable & $P$-value (overall survival) \\
\hline AJCC staging & $<0.0001^{*}$ \\
age $(20<$ vs $\geq 20)$ & 0.2819 \\
Sex & 0.5497 \\
FOXM1 & $0.0302^{*}$ \\
\hline
\end{tabular}

${ }^{*} P<0.05$ by Cox proportional hazards model 


\section{Gene expression analysis in clinical samples}

We conducted a cDNA microarray analysis in 11 frozen samples, and the clustering analysis showed that 32 gene expressions were significantly correlated with FOXM1 expression $(\mathrm{CC}>0.828)$. They are listed in Table 3. The gene ontology (GO) analysis revealed that GO terms that were involved in the cell cycle or mitotic process were enriched in the cluster including FOXM1 (Table 4). Raw data from the microarray analysis are available on the website of the Gene Expression Omnibus (accession no.GSE65532, http:// www.ncbi.nlm.nih.gov/geo/).
Antitumor effect of FOXM1 knockdown in SS cell lines We knocked down FOXM1 in both cell lines by using siRNA. The interruption of FOXM1 was confirmed by Western blotting and TaqMan PCR in both cell lines (Fig. 3a,b). Reduced cell proliferation was recognized only in the SYO-1 cells, not in HS-SY-II (Fig. 3c). Increased sensitivity for DOX was observed in both cell lines by FOXM1 interruption (Fig. 3d).

\section{Antitumor effect of thiostrepton and DOX for SS cell lines} Compared to the untreated controls, decreased FOXM1 expressions were recognized in treated tumor cells by

Table 3 CDNA microarray data analysis: hierarchal cluster of gene expressions correlated with FOXM1 in 11 frozen SS samples (correlation coefficient $>0.828$ )

\begin{tabular}{|c|c|}
\hline Gene symbol & Description \\
\hline FBXO5 & F-box only protein 5 (Early mitotic inhibitor 1) \\
\hline TTK & Dual specificity protein kinase TTK)(Phosphotyrosine picked threonine-protein kinase)(PYT) \\
\hline CENPM & Centromere protein M (CENP-M)(Proliferation-associated nuclear element protein 1) \\
\hline KIF11 & Kinesin-like protein KIF11 (Kinesin-related motor protein Eg5) \\
\hline SGOL2 & Shugoshin-like 2 (Tripin) \\
\hline RBM12 & Copine-1 (Copine I) \\
\hline GINS2 & DNA replication complex GINS protein PSF2 (GINS complex subunit 2) \\
\hline CLSPN & Claspin (hClaspin)(Hu-Claspin) \\
\hline ASF1B & Histone chaperone ASF1B (Anti-silencing function protein 1 homolog B)(hAsf1)(hAsf1b) \\
\hline PRR11 & Proline-rich protein 11 \\
\hline BIRC5 & Baculoviral IAP repeat-containing protein 5 (Apoptosis inhibitor survivin)(Apoptosis inhibitor 4) \\
\hline GTSE1 & G2 and S phase-expressed protein 1 (B99 homolog) \\
\hline C13orf3 & Uncharacterized protein C13orf3 \\
\hline DIAPH3 & Protein diaphanous homolog 3 (Diaphanous-related formin-3)(DRF3) \\
\hline C16orf75 & OB DNA-binding domain-containing protein C16orf175 \\
\hline NCAPD3 & Condensin-2 complex subunit D3 (Non-SMC condensin II complex subunit D3)(hCAP-D3) \\
\hline LMNB2 & Lamin-B2 \\
\hline KIF23 & Kinesin-like protein KIF23 (Mitotic kinesin-like protein 1)(Kinesin-like protein 5) \\
\hline C15orf23 & Putative TRAF4-associated factor 1 \\
\hline $\mathrm{NCAPH}$ & Condensin complex subunit 2 (Non-SMC condensin I complex subunit H)(Barren homolog protein 1) \\
\hline CDCA4 & Cell division cycle-associated protein 4 (Hematopoietic progenitor protein) \\
\hline NUF2 & Kinetochore protein Nuf2 (hsNuf2)(hNuf2)(hNuf2R)(Cell division cycle-associated protein 1) \\
\hline HCAP-G & Condensin complex subunit 3 \\
\hline TOP2A & TOP2A_HUMAN Isoform 2 of P11388 \\
\hline CDCA3 & Cell division cycle-associated protein 3 (Trigger of mitotic entry protein 1)(TOME-1) \\
\hline PBK & Lymphokine-activated killer T-cell-originated protein kinase \\
\hline NCAPG2 & Condensin-2 complex subunit G2 \\
\hline CCNA2 & Cyclin-A2 (Cyclin-A) \\
\hline ZWINT & ZW10 interactor \\
\hline CENPN & Centromere protein N (CENP-N) \\
\hline KNTC2 & Kinetochore protein NDC80 homolog \\
\hline RFC5 & Replication factor C subunit 5 (Activator 1 subunit 5) \\
\hline
\end{tabular}


Table 4 Gene ontology analysis: list of GO terms that were enriched in the cluster including FOXM1 compared with reference genes $(P<5 \times 10-14)$

\begin{tabular}{lll}
\hline Term & Sample frequency (27 genes) & Background frequency (21,804 genes) \\
\hline Mitotic cell cycle (GO:0000278) & 21 & 763 \\
Cell cycle (GO:0007049) & 21 & 1251 \\
Cell cycle process (GO:0022402) & 19 & 972 \\
Mitotic cell cycle process (GO:1903047) & 17 & 685 \\
Nuclear division (GO:0000280) & 15 & 420 \\
Organelle fission (GO:0048285) & 15 & 446 \\
Cell cycle phase (GO:0022403) & 13 & 287 \\
Biological phase (GO:0044848) & 13 & 291 \\
Mitotic nuclear division (GO:0007067) & 13 & 317 \\
M phase (GO:0000279) & 11 & 216 \\
Mitotic M phase (GO:0000087) & 11 & 216 \\
\hline
\end{tabular}

Western blotting (Fig. 4a). Thiostrepton dose-dependently inhibited the cell proliferation for both the SYO-1 and HSSY-II SS cell lines (Fig. 4b). We also evaluated the effect on the proliferation of cell lines treated with thiostrepton, DOX, or their combination. We observed that the cell lines treated with the combination of both drugs showed lower proliferation than those treated with either drug individually (Steel-Dwass multiple comparison test, $P<0.05$ ) (Fig. 4c and d). and TaqMan PCR in both SS cell lines treated with thiostrepton (Additional file 1: Figure S1).

\section{Discussion}

The expression of FOXM1 in clinical specimens has been reported to be an adverse prognostic factor in many malignancies [11, 15-17, 19-21, 24]. In the present study's univariate analysis, FOXM1 expression was revealed to be correlated with poor prognosis for OS and EFS among the SS patients treated with chemotherapy, and the multivariate analysis adjusted for surgical stage, sex and age showed that FOXM1 expression was an independent prognostic factor. Among the clinicopathological factors, high mitotic activity was strongly correlated with overexpression of FOXM1. Immunohistochemically, the MIB-1 labeling index was significantly high in the FOXM1 expression cases.

The cDNA microarray showed that 32 gene expressions were significantly correlated with FOXM1 expression in clinical samples of SS. The GO analysis revealed that many of these genes are involved in the cell cycle and mitosis. Three genes (CCNA2 [34], KIF23 [35] and CDCA3 [36]) involved mainly in controlling late cellcycle events in the $\mathrm{G} 2$ and $\mathrm{M}$ phases were among this group. These three genes have the CHR (cell cycle genes homology region) element in their promoter lesion [37], and FOXM1 controls cell cycle-dependent gene expression through CHR elements [38]. The CHR, typically located at or close to the transcriptional start site of a cluster of genes at the G2-M transition, is coordinated through promoter elements bounded by the dimerization partner, RB-like, E2F and multi-vulval class B (DREAM) and Myb-MuvB (MMB) transcriptional regulatory complexes [38].

Topoisomerase II $\alpha$ and survivin (BIRC5), which are mainly involved in controlling early-phase cell-cycle events, was also shown to correlate with FOXM1 by cDNA microarray. Topoisomerase II $\alpha$ plays a role in mitotic chromosome condensation and segregation, creating double-strand breaks in DNA [39]. In a mouse lung tumogenesis model, FOXM1 directly bound to the topoisomerase II $\alpha$ promoter region [40]. Oda et al. reported that the survival of SS patients with a high expression of topoisomerase II $\alpha$ was worse than that of SS patients with a lower expression [41].

Survivin forms a complex with chromosome passenger proteins Aurora B kinase and inner centromere protein (INCENP), where it plays a critical role in the localization of the Aurora B kinase-INCENP complex to the inner chromosomal region of centromeres at the early stages of mitosis [42]. Interruption of FOXM1 reduced the survivin expression in leukemia [21] and osteosarcoma [25] cell lines and inhibited cell-cycle progression. Survivin also associated with DNA damage response, it may facilitate recruitment of repair proteins at sites of DNA damage and inhibition of survivin mediate the increase chemosensivity for DOX in leukemia cell line [43].

DOX is routinely available for sarcoma treatment in many countries. The first-line chemotherapy for advanced, metastatic or nonresectable soft tissue sarcoma is typically based on DOX as a single agent or in combination with a second drug such as ifosfamide [44].

The results of the present cDNA microarray analysis supported our clinical and pathological finding that FOXM1 expression was correlated with high mitotic activity, a high MIB-1 labeling index and poor prognosis in SS 

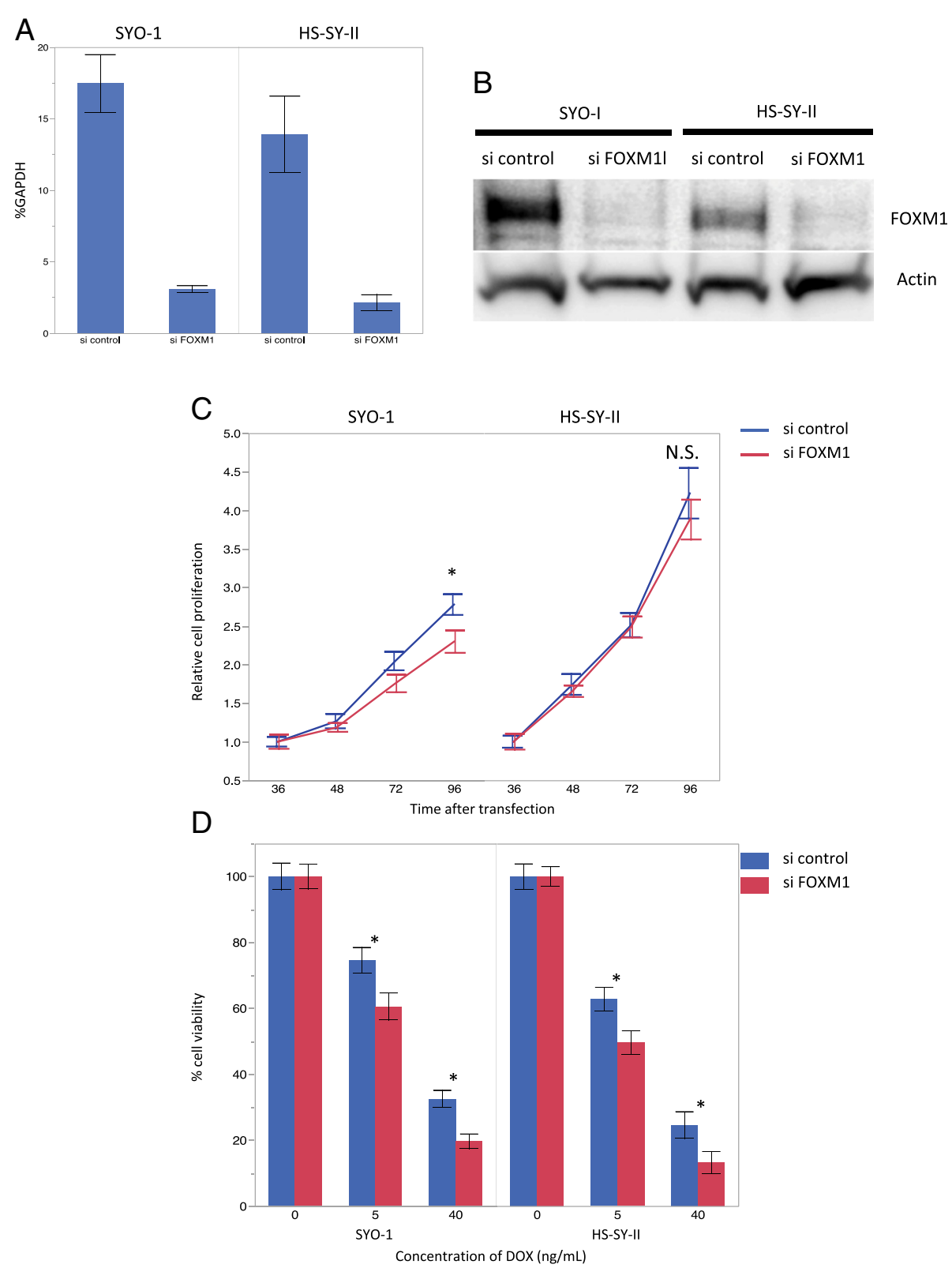

Fig. 3 Proliferation and chemosensitivity assay results in SS cell lines with FOXM1 knockdown. a The cell lines were transduced with FOXM1 siRNA or a nontargeting control. The real-time quantitative PCR for FOXM1 showed a reduction in FOXM1 transcript at $24 \mathrm{~h}$ after transfection. b Western blotting demonstrated that the cell lines transduced with FOXM1 had significantly reduced levels of FOXM1 protein at $48 \mathrm{~h}$ after transfection. c Cell lines with FOXM1 siRNA compared to nontargeting control. Significantly decreased proliferation was recognized in the SYO-1 cells at $96 \mathrm{~h}$ after transfection. $\mathbf{d}$ SiRNA targeting FoxM1 transfected cells had higher sensitivity for DOX, compared with the control. Data are presented as mean $\pm \mathrm{SD}$ for three independent experiments. ${ }^{*} P<0.05$ by by $t$-test. N.S., not significant

patients. These findings indicated that FOXM1 is reliable biomarker for adverse prognosis in SS patients.

FOXM1 interruption by siRNA caused a reduction in cell proliferation, significantly so in the cell line SYO-1. FOXM1 interruption caused decreasing viability treated with DOX in both the SYO-1 and HS-SY-II cells. DOX treatment of cancer cells created double-stranded DNA breaks, and DNA repair genes were induced to rescue the cells from the DNA damage. FOXM1 regulates survivin and other DNA repair genes [45] (XRCC1 and BRCA2) and is involved in chemoresistance via a DNA repair pathway. Other investigators reported that the interruption of FOXM1 expression in breast cancer cells sensitized the cells to DOX [46].

DOX induces acute and chronic toxicities, and treatment options are needed to reduce the dosages of DOX 

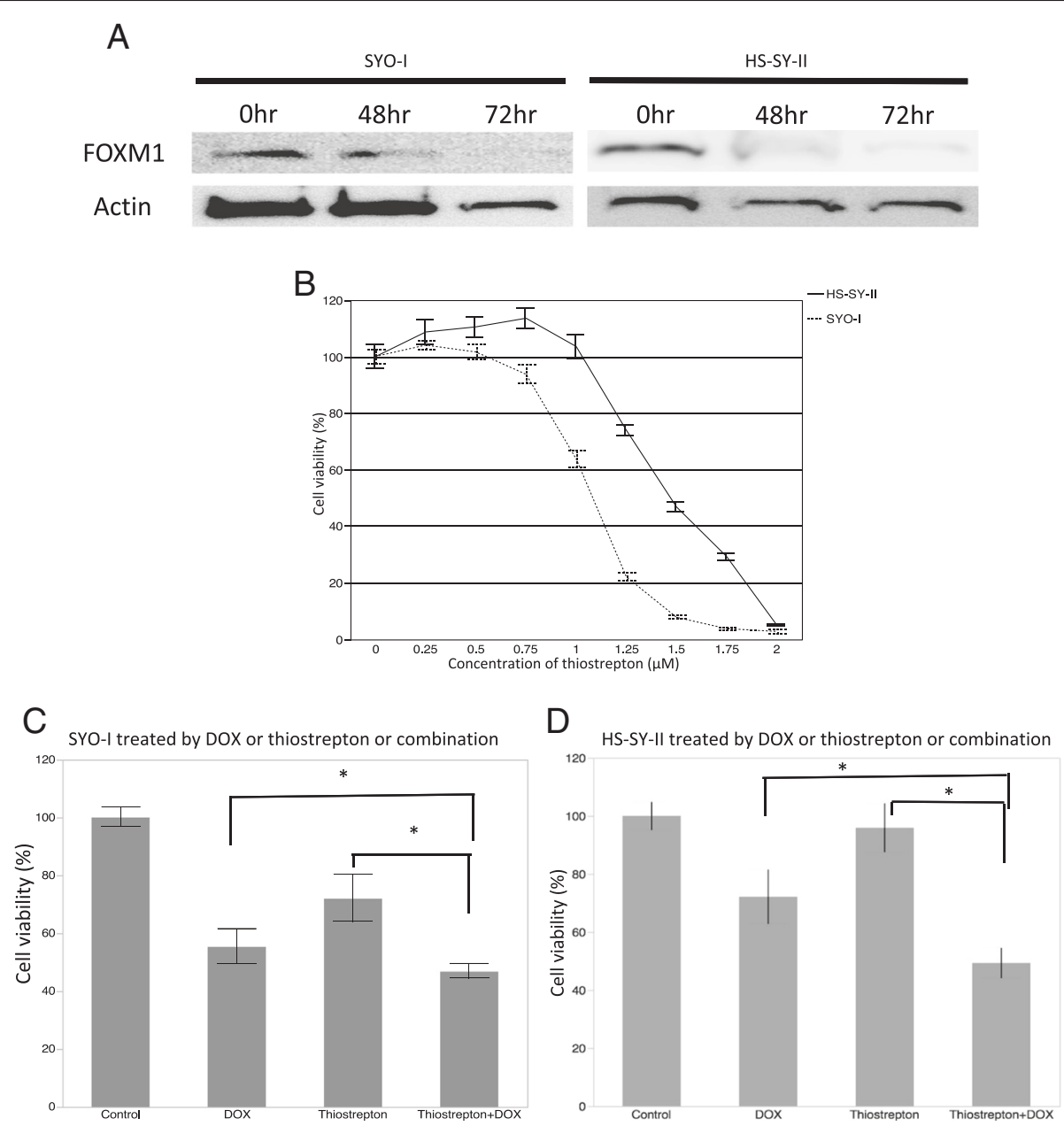

Fig. 4 Thiostrepton reduced FOXM1 expression in the SS cell lines, producing diminished cell viability. a SS cell lines treated with $1 \mu \mathrm{M}$ thiostrepton for $48 \mathrm{~h}$ and $72 \mathrm{~h}$ showed decreased FOXM1 protein on Western blots. b Treatment of SS cell lines with increasing quantities of Thiostrepton for $72 \mathrm{~h}$ resulted in reduced numbers of viable cells compared to diluent controls. $\mathbf{c}$ and $\mathbf{d}$ Proliferation of SS cell lines treated with $1 \mu \mathrm{M}$ thiostrepton, $5 \mathrm{ng} / \mathrm{mL}$ DOX, or their combination. The cell lines treated with the combination of both drugs showed lower proliferation than those treated with each drug individually (c: SYO-I, d: HS-SY-II). ${ }^{*} P<0.05$ by Steel-Dwass multiple comparison test

and enhance its therapeutic efficacy [44]. Here we demonstrated that FOXM1 expression has important roles in cell proliferation and chemoresistance in SS cell lines. We propose that FOXM1 could be a potential therapeutic target for SS.

We also observed that in two SS cell lines, thiostrepton, known as a FOXM1 inhibitor [47], reduced both the number of viable cells in a dose-dependent manner and the levels of FOXM1 protein and mRNA expression. Reduced FOXM1 expression in protein and mRNA levels was recognized at low-toxic concentrations of thiostrepton in the HS-SY-II cells $(1 \mu \mathrm{M})$, and the interruption of FOXM1 could not decrease the cell proliferation in HS-SY-II cells. It was contrary that recognized in SYO-1. This deference effect of FOXM1 interruption between the two cell lines, suggested that the FOXM1 involved in SS tumor progression in a variety of ways.
We supposed that the difference might be due to the morphology (biphasic or monophasic) and genetic basement (SYT-SSX1 or SSX2). But there was no supportive finding by immunohistochemical study and cDNA microarray analysis.

The cytotoxicity in SS cell lines might be not only via the inhibition of FOXM1. The mechanism of FOXM1 interruption by thiostrepton has been proposed to be via the direct binding of FOXM1 [47] and also via its activity as a proteasome inhibitor [48].

Another proteasome inhibitor, bortezomib, also showed the ability to interrupt FOXM1, although there is no evidence of its direct binding to FOXM1 [48]. Little is known about the efficacy of proteasome inhibitors in SS. The proteasome inhibitor MG132 has shown antitumor activity for SS cell lines in vitro [49], However, the results from a Phase II trial of a single use of bortezomib against 
a variety of relapsed or metastatic sarcomas including SS have been discouraging [50]. The efficacy of combination conventional chemotherapy with a proteasome inhibitor against sarcoma has not been established. In our study, both cell lines treated with the combination of thiostrepton and DOX showed lower cellular proliferation than those treated with either drug individually. Thiostrepton has the potential to be a therapeutic agent for SS cases showing FOXM1 expression.

\section{Conclusion}

We have elucidated that FOXM1 inhibition is a candidate treatment option for SS, based on our clinicopathologic assessment and in vitro study, using siRNA and thiostrepton on two SS cell lines. FOXM1 may be involved in SS tumor progression in a variety of ways. Further in vivo and in vitro investigations are warranted to evaluate the efficacy of FOXM1 inhibitors either alone or in combination with other agents.

\section{Additional files}

Additional file 1: SS cell lines treated with $1 \mu \mathrm{M}$ thiostrepton for $48 \mathrm{~h}$ The real-time quantitative PCR showed a reduction of FOXM1 transcript. (PPTX $73 \mathrm{~kb}$ )

\section{Abbreviations}

AJCC, American Joint Committee on Cancer; DOX, doxorubicin; EFS, event-free survival; FNCLCC, French Federation of Cancer Centers; FOXM1, forkhead box M1; HPF, high-power fields; OS, overall survival; SS, synovial sarcoma; WHO, World Health Organization

\section{Acknowledgments}

We thank the Research Support Center, Graduate School of Medical Sciences, Kyushu University for the technical support.

Contract/grant details: This study was supported by a Grant-in-Aid for Scientific Research (B) (25293088) and a Grant-in-Aid for Scientific Research (C) (26462708) from the Japan Society for the Promotion of Science.

\section{Availability of data and materials}

Raw data from the microarray analysis are available on the website of the Gene Expression Omnibus (accession no.GSE65532, http:// www.ncbi.nlm.nih.gov/geo/)

\section{Authors' contributions \\ AM and KK carried out the literature review and drafted the manuscript. MK participated in the development of the methodology and performed the statistical analysis. KI and TI provided technical support about immunohistochemistry and participated in the data discussion, and provided technical support about in situ hybridization and western blotting. ME and TN conceived and supervised in the in vitro study. $Y$ I and $Y O$ was involved in the study design and data discussion, helped to draft the manuscript and gave a final approval of the version to be published. All authors read and approved the final manuscript.}

\section{Competing interests}

The authors declare that they have no competing interests.

\section{Ethics approval and consent to participate}

This study was retrospective study and no individual consent has been obtained from patients. Only anonymized data from our database was used. This study was approved by the Ethics Committee of Kyushu University (No. 26-49).

\section{Author details}

'Department of Anatomic Pathology, Graduate School of Medical Sciences, Kyushu University, 3-1-1 MaidashiHigashi-ku, Fukuoka 812-8582, Japan. ${ }^{2}$ Departments of Orthopedic Surgery, Graduate School of Medical Sciences, Kyushu University, Fukuoka, Japan. ${ }^{3}$ Division of Cancer Immunotherapy, National Cancer Center Hospital East, Kashiwa, Japan.

Received: 26 July 2015 Accepted: 8 July 2016

Published online: 20 July 2016

\section{References}

1. Weiss SW, Goldblum JR. Enzinger and Weiss's soft tissue tumors. 5th ed. St. Louis: Mosby Inc; 2008. p. 1161-82.

2. Fligman I, Lonardo F, Jhanwar SC, Gerald WL, Woodruff J, Ladanyi M. Molecular diagnosis of synovial sarcoma and characterization of a variant SYT-SSX2 fusion transcript. Am J Pathol. 1995;147:1592-9.

3. Ferrari A, Bisogno G, Alaggio R, Cecchetto G, Collini P, Rosolen A, et al. Synovial sarcoma of children and adolescents: the prognostic role of axial sites. Eur J Cancer. 2008;44:1202-9.

4. Palmerini E1, Staals EL, Alberghini M, Zanella L, Ferrari C, Benassi MS, et al. Synovial sarcoma: retrospective analysis of 250 patients treated at a single institution. Cancer. 2009:115:2988-98.

5. Sultan I, Rodriguez-Galindo C, Saab R, Yasir S, Casanova M, Ferrari A. Comparing children and adults with synovial sarcoma in the surveillance, epidemiology, and end results program, 1983 to 2005: an analysis of 1268 patients. Cancer. 2009:115:3537-47.

6. Italiano A, Penel N, Robin YM, Bui B, Le Cesne A, Piperno-Neumann S, et al. Neo/adjuvant chemotherapy does not improve outcome in resected primary synovial sarcoma: a study of the French Sarcoma Group. Ann Oncol. 2009;20:425-30.

7. Korver W, Roose J, Clevers $\mathrm{H}$. The winged-helix transcription factor Trident is expressed in cycling cells. Nucleic Acids Res. 1997;25:1715-9.

8. Yao KM, Sha M, Lu Z, Wong GG. Molecular analysis of a novel winged helix protein, WIN. Expression pattern, DNA binding property, and alternative splicing within the DNA binding domain. J Biol Chem. 1997;272:19827-36.

9. Wonsey DR, Follettie MT. Loss of the forkhead transcription factor FoxM1 causes centrosome amplification and mitotic catastrophe. Cancer Res. 2005;65:5181-9.

10. Koo C-Y, Muir KW, La EWF. Review FOXM1: from cancer initiation to progression and treatment. Biochim Biophys Acta. 1819;2012:28-37.

11. Yang DK, Son CH, Lee SK, Choi PJ, Lee KE, Roh MS. Forkhead box M1 expression in pulmonary squamous cell carcinoma: correlation with clinicopathologic features and its prognostic significance. Hum Pathol. 2009; 40:464-70.

12. Ha SY, Lee $\mathrm{CH}$, Chang HK, Chang S, Kwon KY, Lee EH, Roh MS, Seo B. Differential expression of forkhead box $\mathrm{M} 1$ and its downstream cyclindependent kinase inhibitors p27 (kip1) and p21 (waf1/cip1) in the diagnosis of pulmonary neuroendocrine tumours. Histopathology. 2012;60(5):731-9.

13. Teh MT, Wong ST, Neill GW, Ghali LR, Philpott MP, Quinn AG. FOXM1 is a downstream target of Gli1 in basal cell carcinomas. Cancer Res. 2002;62:4773-80.

14. Ahmad A, Wang Z, Kong D, Ali S, Li Y, Banerjee S, et al. FoxM1 downregulation leads to inhibition of proliferation, migration and invasion of breast cancer cells through the modulation of extra-cellular matrix degrading factors. Breast Cancer Res Treat. 2010;122:337-46.

15. Huang C, Qiu Z, Wang L, Peng Z, Jia Z, Logsdon CD, et al. A novel FoxM1caveolin signaling pathway promotes pancreatic cancer invasion and metastasis. Cancer Res. 2012;72:655-65.

16. Chu XY, Zhu ZM, Chen LB, Wang JH, Su QS, Yang JR, et al. FOXM1 expression correlates with tumor invasion and a poor prognosis of colorectal cancer. Acta Histochem. 2012;114:755-62.

17. Priller M, Pöschl J, Abrão L, von Bueren AO, Cho YJ, Rutkowski S, et al. Expression of FoxM1 is required for the proliferation of medulloblastoma cells and indicates worse survival of patients. Clin Cancer Res. 2011;17:6791-801.

18. Kim IM, Ackerson T, Ramakrishna S, Tretiakova M, Wang IC, Kalin TV, et al. The Forkhead box $\mathrm{m} 1$ transcription factor stimulates the proliferation of tumor cells during development of lung cancer. Cancer Res. 2006;66:2153-61.

19. Wang Y, Wen L, Zhao SH, Ai ZH, Guo JZ, Liu WC. FoxM1 expression is significantly associated with cisplatin-based chemotherapy resistance and poor prognosis in advanced non-small cell lung cancer patients. Lung Cancer. 2013;79:173-9. 
20. Li X, Qi W, Yao R, Tang D, Liang J. Overexpressed transcription factor FOXM1 is a potential diagnostic and adverse prognostic factor in postoperational gastric cancer patients. Clin Transl Oncol. 2014;16:307-14.

21. Nakamura S, Hirano I, Okinaka K, Takemura T, Yokota D, Ono T, et al. The FOXM1 transcriptional factor promotes the proliferation of leukemia cells through modulation of cell cycle progression in acute myeloid leukemia. Carcinogenesis. 2010;31:2012-21.

22. Wan X, Yeung C, Kim SY, Dolan JG, Ngo VN, Burkett S, et al. Identification of FoxM1/Bub1b signaling pathway as a required component for growth and survival of rhabdomyosarcoma. Cancer Res. 2012;72:5889-99.

23. Christensen L, Joo J, Lee $S$, Wai $D$, Triche TJ, May WA. FOXM1 is an oncogenic mediator in Ewing sarcoma. PLoS One. 2013;8:e54556.

24. Yu J, Deshmukh H, Payton JE, Dunham C, Scheithauer BW, Tihan T, et al. Array-based comparative genomic hybridization identifies CDK4 and FOXM1 alterations as independent predictors of survival in malignant peripheral nerve sheath tumor. Clin Cancer Res. 2011;17:1924-34.

25. Wang IC, Chen YJ, Hughes D, Petrovic V, Major ML, Park HJ, et al. Forkhead box $\mathrm{M} 1$ regulates the transcriptional network of genes. Essential for mitotic progression and genes encoding the SCF (Skp2-Cks1) ubiquitin ligase. Mol Biol Cell. 2005;25:10875-94.

26. Grant GD, Brooks 3rd L, Zhang X, Mahoney JM, Martyanov V, Wood TA, et al. Identification of cell cycle-regulated genes periodically expressed in U2OS cells and their regulation by FOXM1 and E2F transcription factors. Mol Biol Cell. 2013;24(23):3634-50.

27. Halasi M, Gartel AL. Suppression of FOXM1 sensitizes human cancer cells to cell death induced by DNA-damage. PLoS One. 2012;7(2):e31761.

28. Fletcher CD, Bridge AJ, Hogendoom PC, editors. World Health Organization classification of tumours. Pathology and genetics of tumours of soft tissue and bone. Lyon: IARC Press; 2013. p. 213-5.

29. Edge SB, Byrd DR, Compton CC, Fritz AG, Greene FL, Trotti A. AJCC cancer staging manual. 7th ed. St. Louis: Springer; 2010.

30. Kawai A, Naito N, Yoshida A, Morimoto Y, Ouchida M, Shimizu K, et al. Establishment and characterization of a biphasic synovial sarcoma cell line, SYO-1. Cancer Lett. 2004;204(1):105-13.

31. Sonobe H, Manabe Y, Furihata M, Iwata J, Oka T, Ohtsuki Y, et al. Establishment and characterization of a new human synovial sarcoma cell line, HS-SY-II. Lab Invest. 1992;67(4):498-505.

32. Jin L, Majerus J, Oliveira A, Inwards CY, Nascimento AG, Burgart $L$, et al. Detection of fusion gene transcripts in fresh-frozen and formalin-fixed paraffin-embedded tissue sections of soft-tissue sarcomas after laser capture microdissection and rt-PCR. Diagn Mol Pathol. 2003;12:224-30.

33. Endo $M$, Yamamoto $H$, Setsu $N$, Kohashi $K$, Takahashi $Y$, Ishii $T$, et al. Prognostic significance of AKT/mTOR and MAPK pathways and antitumor effect of mTOR inhibitor in NF1-related and sporadic malignant peripheral nerve sheath tumors. Clin Cancer Res. 2012;15(19):450-61.

34. Zwicker J, Lucibello FC, Wolfraim LA, Gross C, Truss M, Engeland K, et al. Cell cycle regulation of the cyclin $\mathrm{A}, \mathrm{cdc} 25 \mathrm{C}$ and cdc2 genes is based on a common mechanism of transcriptional repression. EMBO J. 1995;14:4514-22.

35. Seguin L, Liot C, Mzali R, Harada R, Siret A, Nepveu A, et al. CUX1 and E2F1 regulate coordinated expression of the mitotic complex genes Ect2, MgcRac GAP, and MKLP1 in S phase. Mol Cell Biol. 2009;29:570-81.

36. Yoshida K. Cell-cycle-dependent regulation of the human and mouse Tome-1 promoters. FEBS Lett. 2005;579:1488-92.

37. Muller GA, Engeland K. The central role of CDE/CHR promoter elements in the regulation of cell cycle-dependent gene transcription. FEBS J. 2010; 277(4):877-93.

38. Chen X, Müller GA, Quaas M, Fischer M, Han N, Stutchbury B, et al. The forkhead transcription factor FOXM1 controls cell cycle-dependent gene expression through an atypical chromatin binding mechanism. Mol Cell Biol. 2013;33:227-36

39. Osheroff N, Zechiedrich EL, Gale KC. Catalytic function of DNA topoisomerase II. Bioessays. 1991;13:269-75.

40. Wang IC, Meliton L, Ren X, Zhang Y, Balli D, Snyder J, et al. Deletion of Forkhead Box M1 transcription factor from respiratory epithelial cells inhibits pulmonary tumorigenesis. PLoS One. 2009;4(8):e6609.

41. Oda Y, Ohishi $Y$, Saito T, Hinoshita E, Uchiumi T, Kinukawa N, et al. Nuclear expression of Y-box-binding protein-1 correlates with P-glycoprotein and topoisomerase II alpha expression, and with poor prognosis in synovial sarcoma. J Pathol. 2003;199(2):251-8.

42. Bolton MA, Lan W, Powers SE, McCleland ML, Kuang J, Stukenberg PT. Aurora B kinase exists in a complex with survivin and INCENP and its kinase activity is stimulated by survivin binding and phosphorylation. Mol Biol Cell. 2002;13:3064-77.

43. Huang J, Lyu H, Wang J, Liu B. Influence of survivin-targeted therapy on chemosensitivity in the treatment of acute myeloid leukemia. Cancer Lett. 2015;366(2):160-72.

44. Schöffski P, Cornillie J, Wozniak A, Li H, Hompes D. Soft tissue sarcoma: an update on systemic treatment options for patients with advanced disease. Oncol Res Treat. 2014;37:355-62.

45. Tan Y, Raychaudhuri P, Costa RH. Chk2 mediates stabilization of the FoxM1 transcription factor to stimulate expression of DNA repair genes. Mol Cell Biol. 2007;27:1007-16.

46. Park YY, Jung SY, Jennings NB, Rodriguez-Aguayo C, Peng G, Lee SR, et al. FOXM1 mediates Dox resistance in breast cancer by enhancing DNA repair. Carcinogenesis. 2012;33(10):1843-53.

47. Hegde NS, Sanders DA, Rodriguez R, Balasubramanian S. The transcription factor FOXM1 is a cellular target of the natural product thiostrepton. Nat Chem. 2011;3:725-31.

48. Bhat UG, Halasi M, Gartel AL. FoxM1 is a general target for proteasome inhibitors. PLoS One. 2009;4:e6593.

49. Cheong $\mathrm{HJ}$, Lee KS, Woo IS, Won JH, Byun JH. Up-regulation of the DR5 expression by proteasome inhibitor MG132 augments TRAIL-induced apoptosis in soft tissue sarcoma cell lines. Cancer Res Treat. 2011:43(2):124-30.

50. Maki RG, Kraft AS, Scheu K, Yamada J, Wadler S, et al. A multicenter phase II study of Bortezomib in recurrent or metastatic sarcomas. Cancer. 2005;103:1431-8.

\section{Submit your next manuscript to BioMed Central and we will help you at every step:}

- We accept pre-submission inquiries

- Our selector tool helps you to find the most relevant journal

- We provide round the clock customer support

- Convenient online submission

- Thorough peer review

- Inclusion in PubMed and all major indexing services

- Maximum visibility for your research

Submit your manuscript at www.biomedcentral.com/submit
Biomed Central 